\title{
The Solution of a Class of Third-Order Boundary Value Problems by the Reproducing Kernel Method
}

\author{
Zhiyuan Li, ${ }^{1}$ Yulan Wang, ${ }^{1}$ and Fugui Tan ${ }^{2}$ \\ ${ }^{1}$ Department of Mathematics, Inner Mongolia University of Technology, Hohhot 010051, China \\ 2 Jining Teachers College, Jining 012000, China \\ Correspondence should be addressed to Yulan Wang, wylnei@163.com
}

Received 1 July 2012; Accepted 20 September 2012

Academic Editor: Wing-Sum Cheung

Copyright (C) 2012 Zhiyuan Li et al. This is an open access article distributed under the Creative Commons Attribution License, which permits unrestricted use, distribution, and reproduction in any medium, provided the original work is properly cited.

This paper expands the application of reproducing kernel method to a class of third-order boundary value problems with mixed nonlinear boundary conditions. The analytical solution is represented in the form of series in the reproducing kernel space. The $n$-term approximation is obtained and is proved to converge to the analytical solution. The numerical examples are given to demonstrate the computation efficiency of the presented method. Results obtained by the method indicate that the method is simple and effective.

\section{Introduction}

Third-order differential equations arise in a variety of different areas of applied mathematics and physics, for example, in the deflection of a curved beam having a constant or varying cross section, a three layer beam, electromagnetic waves, or gravity driven flows. Third-order boundary value problems were discussed in many papers in recent years, for instance, see [16] and references therein. In [1-3], the authors used the spline functions to solve boundary value problems. In [4], the authors developed a second-order method for solving third-order three-point boundary value problems based on Padé approximant in a recurrence relation. In [5], the authors introduced Adomian decomposition method for multipoint boundary value problems (BVPs). In this paper, we use reproducing kernel to solve singular third-order boundary value problems with mixed boundary conditions. Recently, the reproducing kernel methods [7-10] emerge one after another. Using the reproducing kernel methods, the authors discussed two-point boundary value problems and periodic boundary value problems. For third-order boundary value problems with mixed nonlinear boundary conditions, however, this method has not yet been applied. In previous work, the reproducing kernel method cannot be used directly to solve third-order boundary value problems with nonlinear 
boundary conditions. Our work is to present a numerical algorithm for solving a class of singular third-order boundary value problems. By using this method, the analytical solution and approximate solution are given and uniformly converge to the exact solution and its corresponding derivatives. The algorithms are efficiently applied to solve some model problems. equations:

Let us consider the following singular problems of third-order ordinary differential

$$
\begin{gathered}
u^{\prime \prime \prime}(x)+p_{1}(x) u^{\prime \prime}(x)+p_{2}(x) u^{\prime}(x)+p_{3}(x) u(x)=F(x), \quad x \in(0,1), \\
\lambda_{i} u=r_{i}, \quad(i=1,2,3),
\end{gathered}
$$

where $p_{j}(x), f(x) \in L^{2}[0,1],(j=1,2,3)$ are known functions. $\lambda_{i} u,(i=1,2,3)$ are linear independence conditions of determining the solution. We assume that (1.1) has a unique solution which belongs to $W_{2}^{4}[0,1]$, where $W_{2}^{4}[0,1]$ which is a reproducing kernel space is defined in the second section.

In order to solve (1.1), let $L u=u^{\prime \prime \prime}(x)+p_{1}(x) u^{\prime \prime}(x)+p_{2}(x) u^{\prime}(x)+p_{3}(x) u(x)$. It is easy to prove that $L: W_{2}^{4}[0,1] \rightarrow L^{2}[0,1]$ is bounded linear operator.On the other hand, we assume that the conditions of determining the solution can be homogenized; after homogenization of these conditions, we put the conditions into the reproducing kernel space $W_{2}^{4}[0,1]$ constructed in the following section. Equation (1.1) can be transformed into the following form in $W_{2}^{4}[0,1]$ :

$$
(L u)(x)=F(x) .
$$

To solve problem (1.2), we give a space $W_{2}^{4}[0,1]=\left\{u \mid u \in \bar{W}_{2}^{4}[0,1]\right.$ and $\left.\lambda_{i} u=0, i=1,2,3\right\}$. The inner product in $W_{2}^{4}[0,1]$ is given by $\langle u(x), v(x)\rangle=\sum_{i=0}^{3} u^{(i)}(0) v^{(i)}(0)+\int_{0}^{1} u^{(4)}(t) v^{(4)}(t) d t$. Like in [8], we can get the following reproducing kernel space.

Theorem 1.1. The space $W_{2}^{4}[0,1]$ is a reproducing kernel space and its reproducing kernel is $K(x, y)$, and

$$
K(x, y)=R_{y}(x)-\frac{h_{1}(x) h_{1}(y)}{\left\|h_{1}(x)\right\|^{2}}-\frac{h_{2}(x) h_{2}(y)}{\left\|h_{2}(x)\right\|^{2}}-\frac{h_{3}(x) h_{3}(y)}{\left\|h_{3}(x)\right\|^{2}}
$$

where

$$
R_{y}(x)= \begin{cases}\frac{5040-y^{7}+35 x^{3} y^{3}(4+y)-21 x^{2} y^{2}\left(-60+y^{3}\right)+7 x y\left(720+y^{5}\right)}{5040}, & x<y \\ \frac{5040-x^{7}+5040 x y+7 x^{6} y+1260 x^{2} y^{2}-21 x^{5} y^{2}+140 x^{3} y^{3}+35 x^{4} y^{3}}{5040}, & y<x\end{cases}
$$

$h_{1}(x)=\lambda_{1 y} R_{y}(x), h_{2}(x)=\lambda_{2 y}\left(R(x, y)-\left(h_{1}(x) h_{1}(y) /\left\|h_{1}(x)\right\|^{2}\right)\right), h_{3}(x)=\lambda_{3 y}(R(x, y)-$ $\left.\left(h_{1}(x) h_{1}(y) /\left\|h_{1}(x)\right\|^{2}\right)-\left(h_{2}(x) h_{2}(y) /\left\|h_{2}(x)\right\|^{2}\right)\right)$, the symbol $\lambda_{i y}$ indicates that the operator $\lambda_{i}$ applies to the function of $y$. 


\section{The Reproducing Kernel Method}

Let $\psi_{i}(x)=\left(L_{y} K_{x}(y)\right)\left(x_{i}\right), i=1,2, \ldots$, Practise Gram-Schmidt orthonomalization for $\left\{\psi_{i}(x)\right\}_{i=1}^{\infty}$, we get

$$
\bar{\psi}_{i}(x)=\sum_{k=1}^{i} \beta_{i k} \psi_{k}(x)
$$

where $\beta_{i k}$ are coefficients of Gram-Schmidt orthonormalization.

Theorem 2.1. If $\left\{x_{i}\right\}_{i=1}^{\infty}$ is distinct points dense in $[0,1]$ and $L^{-1}$ is existent, then

$$
u(x)=\sum_{i=1}^{\infty} \sum_{k=1}^{i} \beta_{i k} F\left(x_{k}\right) \bar{\psi}_{i}(x)
$$

is an analytical solution of the problem (1.2).

Proof. Since $\left\{\bar{\psi}_{i}(x)\right\}_{i=1}^{\infty}$ is an orthonormal systems, $u(x)$ is expressed as

$$
\begin{aligned}
u(x) & =\sum_{i=1}^{\infty}\left\langle u(x), \bar{\psi}_{i}(x)\right\rangle \bar{\psi}_{i}(x) \\
& =\sum_{i=1}^{\infty} \sum_{k=1}^{i} \beta_{i k}\left\langle u(x),\left(L_{s} K_{x}(s)\right)\left(x_{k}\right)\right\rangle \bar{\psi}_{i}(x) \\
& =\sum_{i=1}^{\infty} \sum_{k=1}^{i} \beta_{i k}\left(L_{s}\left\langle u(x), K_{x}(s)\right\rangle\right)\left(x_{k}\right) \bar{\psi}_{i}(x) \\
& =\sum_{i=1}^{\infty} \sum_{k=1}^{i} \beta_{i k}\left(L_{s} u(s)\right)\left(x_{k}\right) \bar{\psi}_{i}(x) \\
& =\sum_{i=1}^{\infty} \sum_{k=1}^{i} \beta_{i k} F\left(x_{k}\right) \bar{\psi}_{i}(x) .
\end{aligned}
$$

We denote the approximate solution of $u_{n}(x)$ by

$$
u_{n}(x)=\sum_{i=1}^{n} \sum_{k=1}^{i} \beta_{i k} F\left(x_{k}\right) \bar{\psi}_{i}(x)
$$

Theorem 2.2. Let $\varepsilon_{n}^{2}=\left\|u(x)-u_{n}(x)\right\|^{2}$ where $u(x), u_{n}(x)$ are given by (2.2) and (2.4), then the sequence of number $\varepsilon_{n}(x)$ is monotone decreasing and $\varepsilon_{n}(x) \rightarrow 0$. 
Proof. Because

$$
\varepsilon_{n}^{2}=\left\|u(x)-u_{n}(x)\right\|^{2}=\sum_{i=n+1}^{\infty}\left\langle u(x), \bar{\psi}_{i}(x)\right\rangle \bar{\psi}_{i}(x)=\sum_{i=n+1}^{\infty}\left(\left\langle u(x), \bar{\psi}_{i}(x)\right\rangle\right)^{2},
$$

clearly $\varepsilon_{n-1} \geq \varepsilon_{n}$ and consequently $\left\{\varepsilon_{n}\right\}$ is monotone decreasing in the sense of $\|\cdot\|_{W_{2}^{4}}$. By Theorem 2.1, we know $\sum_{i=1}^{\infty}\left\langle u(x), \bar{\psi}_{i}(x)\right\rangle \bar{\psi}_{i}(x)$ is convergent in the norm of $\|\cdot\|_{W_{2}^{4}}$, then we have

$$
\varepsilon_{n}^{2}=\left\|u(x)-u_{n}(x)\right\|^{2} \longrightarrow 0 .
$$

Hence, $\varepsilon_{n} \rightarrow 0$.

Theorem 2.3 (convergence analysis). If $u(x), u_{n}(x)$ are given by (2.2) and (2.4), then $u_{n}(x)$ and $u_{n}^{(k)}(x)$ uniformly convergent to $u(x)$ and $u^{(k)}(x)$, where $k=0,1,2,3$.

Proof. For any $x \in[0,1], k=0,1,2,3$

$$
\left|u_{n}^{(k)}(x)-u^{(k)}(x)\right|=\left|\left\langle u_{n}(t)-u(t), \frac{\partial^{k} K(x, t)}{\partial x^{k}}\right\rangle\right| \leq\left\|u_{n}(t)-u(t)\right\| \cdot\left\|\frac{\partial^{k} K(x, t)}{\partial x^{k}}\right\|,
$$

then there exists $C_{k}>0$ such that, $\left|u_{n}^{(k)}(x)-u^{(k)}(x)\right| \leq C_{k}\left\|u_{n}(t)-u(t)\right\|=C_{k} \varepsilon_{n} \rightarrow 0$.

Theorem 2.4. If $\left\{x_{k}\right\}_{k=1}^{\infty}$ is distinct points dense in $[0,1]$ and $u(x), u_{n}(x)$ are given by (2.2) and (2.4), then $\operatorname{Lu}\left(x_{k}\right)=L u_{n}\left(x_{k}\right)$.

Proof. We may set projective operator $P_{n}: W_{2}^{4}[0,1] \rightarrow\left\{\sum_{m=1}^{n} c_{m} \psi_{m}(x), c_{m} \in R\right\}$. Hence,

$$
\begin{aligned}
L u_{n}\left(x_{k}\right) & =\left\langle u_{n}(\xi), L_{x_{k}} K_{x_{k}}(\xi)\right\rangle=\left\langle u_{n}(\xi), \psi_{k}(\xi)\right\rangle=\left\langle P_{n} u(\xi), \psi_{k}(\xi)\right\rangle \\
& =\left\langle u(\xi), P_{n} \psi_{k}(\xi)\right\rangle=\left\langle u(\xi), \psi_{k}(\xi)\right\rangle=\left\langle u(\xi), L_{x_{k}} K_{x_{k}}(\xi)\right\rangle \\
& =L_{x_{k}}\left\langle u(\xi), K_{x_{k}}(\xi)\right\rangle=L_{x_{k}} u\left(x_{k}\right)=L u\left(x_{k}\right) .
\end{aligned}
$$

Theorem 2.5 (error estimate). If $\left\{x_{k}\right\}_{k=1}^{\infty}$ is distinct points dense in $[0,1]$ and $u(x), u_{n}(x)$ are given by (2.2) and (2.4), then $\left|u(x)-u_{n}(x)\right|<(M / n)$, where $M=\left\|\sum_{i=n+1}^{\infty} \sum_{k=1}^{i} \beta_{i k} F\left(x_{k}\right) \bar{\psi}_{i}(x)\right\|$. $\left\|\partial K_{\eta}(\xi) / \partial \eta\right\|$.

Proof. For every given $x \in[0,1]$, there is always $x_{i} \in\left\{x_{k}\right\}_{k=1}^{\infty}$ satisfying $x_{i}<x$ and $x-x_{i}=$ $1 / n$. By Theorem 2.5 and $x_{i} \in\left\{x_{k}\right\}_{k=1}^{\infty}$ implying $L u\left(x_{i}\right)=L u_{n}\left(x_{i}\right)$, so we obtain

$$
\left|L u(x)-L u_{n}(x)\right|=\left|L u(x)-L u\left(x_{i}\right)-\left[L u_{n}(x)-L u_{n}\left(x_{i}\right)\right]\right| .
$$


For application reproducing kernel property, we have

$$
u(x)=\left\langle u(\xi), K_{x}(\xi)\right\rangle, \quad L u(x)=\left\langle u(\xi), L K_{x}(\xi)\right\rangle .
$$

We also have

$$
\begin{aligned}
L u(x)-L u_{n}(x) & =L u(x)-L u\left(x_{i}\right)-\left[L u_{n}(x)-L u_{n}\left(x_{i}\right)\right] \\
& =\left\langle u(\xi), L K_{x}(\xi)-L K_{x_{i}}(\xi)\right\rangle-\left\langle u_{n}(\xi), L K_{x}(\xi)-L K_{x_{i}}(\xi)\right\rangle \\
& =\left\langle u(\xi)-u_{n}(\xi), L K_{x}(\xi)-L K_{x_{i}}(\xi)\right\rangle .
\end{aligned}
$$

Moreover,

$$
\begin{aligned}
\left|u(x)-u_{n}(x)\right| & =\left|L^{-1}\left[L u(x)-L u_{n}(x)\right]\right| \\
& \leq\left|\left\langle u(\xi)-u_{n}(\xi), L^{-1} L K_{x}(\xi)-L^{-1} L K_{x_{i}}(\xi)\right\rangle\right| \\
& =\left|\left\langle u(\xi)-u_{n}(\xi), K_{x}(\xi)-K_{x_{i}}(\xi)\right\rangle\right| \\
& \leq\left\|u-u_{n}\right\|\left\|K_{x}(\xi)-K_{x_{i}}(\xi)\right\| .
\end{aligned}
$$

It is noted that we take norm of $\left\|K_{x}(\xi)-K_{x_{i}}(\xi)\right\|$ for variable $\xi$. The function $k_{x}(\xi)$ is derived on $x$ in the interval of $[0,1]$, so we have $K_{x}(\xi)-K_{x_{i}}(\xi)=\left(\partial K_{\eta}(\xi) / \partial \eta\right)\left(x-x_{i}\right)$. Hence,

$$
\begin{aligned}
\left|u(x)-u_{n}(x)\right| & \leq\left\|u-u_{n}\right\|\left\|\frac{\partial K_{\eta}(\xi)}{\partial \eta}\left(x-x_{i}\right)\right\| \\
& =\left\|u-u_{n}\right\|\left\|\frac{\partial K_{\eta}(\xi)}{\partial \eta}\right\|\left(x-x_{i}\right)=\left\|\sum_{i=n+1}^{\infty} \sum_{k=1}^{i} \beta_{i k} F\left(x_{k}\right) \bar{\psi}_{i}(x)\right\|\left\|\frac{\partial K_{\eta}(\xi)}{\partial \eta}\right\|\left(x-x_{i}\right) \leq \frac{M}{n} .
\end{aligned}
$$

\section{Numerical Experiment}

For showing the effectiveness of our method, we consider the following problems.

Example 3.1 (see $[2,3]$ ). Considering the following third-order boundary values problem

$$
\begin{gathered}
u^{\prime \prime \prime}(x)-x u(x)=\left(x^{3}-2 x^{2}-5 x-3\right) e^{x}, \quad 0 \leq x \leq 1, \\
u(0)=u(1)=0, \quad u^{\prime}(0)=1,
\end{gathered}
$$


where the exact solution is $u_{T}(x)=x(1-x) e^{x}$. By the homogeneous process of the boundary condition, let $v(x)=u(x)-x(1-x)$, problem (3.8) can be transformed into the equivalent form

$$
\begin{gathered}
v^{\prime \prime \prime}(x)-x v(x)=(1-x) x^{2}+\left(x^{3}-2 x^{2}-5 x-3\right) e^{x}, \quad 0 \leq x \leq 1 \\
v(0)=v(1)=0, \quad v^{\prime}(0)=0 .
\end{gathered}
$$

The numerical results are presented in Tables 1, 2, and 3.

Example 3.2 (see $[11,14]$ ). Considering the following third-order obstacle problems:

$$
u^{\prime \prime \prime}(x)= \begin{cases}0, & 0 \leq x \leq \frac{1}{4}, \frac{1}{4} \leq x \leq \frac{1}{4} \\ u(x)-1, & \frac{3 \pi}{4} \leq x \leq 1\end{cases}
$$

where $u(0)=u^{\prime}(0)=u^{\prime}(1)=0$, the exact solution is

$$
u_{T}(x)= \begin{cases}\frac{1}{2} a_{1} x^{2}, & 0 \leq x \leq \frac{1}{4}, \\ 1+a_{2} e^{x}+e^{-x / 2}\left[a_{3} \cos \frac{\sqrt{3} x}{2}+a_{4} \sin \frac{\sqrt{3} x}{2}\right], & \frac{1}{4} \leq x \leq \frac{3}{4} \\ \frac{1}{2} a_{5} x(x-2)+a_{6}, & \frac{3}{4} \leq x \leq 1,\end{cases}
$$

where

$$
\begin{aligned}
& a_{1}=0.24391096222648, \quad a_{2}=-0.17847234452746, \quad a_{3}=-0.81893573565615, \\
& a_{4}=-0.30266818001866, \quad a_{5}=-0.24213890868443, \quad a_{6}=-0.06537630092111 \text {. }
\end{aligned}
$$

The numerical results are presented in Table 4.

Example 3.3. Considering the following boundary value problems with nonclassical condition:

$$
\begin{gathered}
u^{\prime \prime \prime}(x)+\frac{1}{x \sqrt{1-x}} u^{\prime \prime}(x)+\frac{1}{x \sqrt{x(1-x)}} u^{\prime}(x)+\frac{1}{x^{2} \sqrt{1-x}} u(x)=f(x), \quad 0<x<1, \\
u(0)=0, \quad u^{\prime}(0)=\int_{0}^{1} u(x) d x, \quad 2 u(1)=u^{\prime}(1) .
\end{gathered}
$$

We determine $f(x)$ to get the true solution, given by $u_{T}(x)=x e^{x}$. The numerical results are presented in Table 5. 


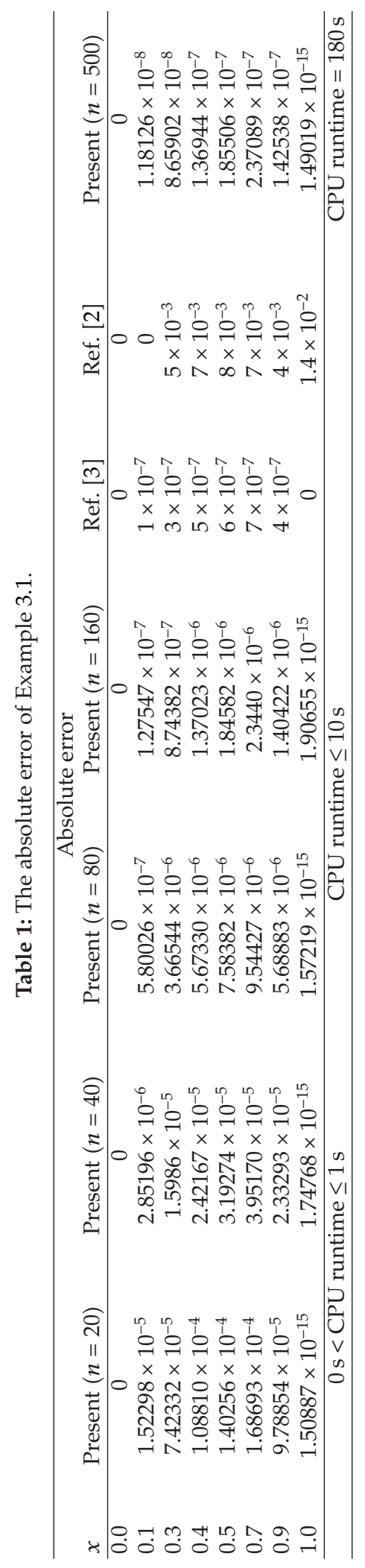


Table 2: The numerical results of Example 3.1.

\begin{tabular}{lcccccc}
\hline$x$ & $u_{T}^{\prime}(x)$ & $u_{500}^{\prime}(x)$ & $\left|u_{T}^{\prime}(x)-u_{500}^{\prime}(x)\right|$ & $u_{T}^{\prime \prime}(x)$ & $u_{500}^{\prime \prime}(x)$ & $\left|u_{T}^{\prime \prime}(x)-u_{500}^{\prime \prime}(x)\right|$ \\
\hline 0 & 1 & 1 & 0 & 0 & 0.0000123873 & 0.0000123873 \\
0.1 & 0.983602 & 0.983602 & $2.217025 \times 10^{-7}$ & -0.342603 & -0.342601 & $1.904429 \times 10^{-6}$ \\
0.3 & 0.823414 & 0.823414 & $4.842037 \times 10^{-7}$ & -1.33636 & -1.33636 & $6.462399 \times 10^{-7}$ \\
0.5 & 0.41218 & 0.412181 & $4.459369 \times 10^{-7}$ & -2.88526 & -2.88526 & $1.127018 \times 10^{-6}$ \\
0.7 & -0.382613 & -0.382613 & $1.172353 \times 10^{-8}$ & -5.21562 & -5.21562 & $3.578872 \times 10^{-6}$ \\
0.9 & -1.74632 & -1.74632 & $1.0452710 \times 10^{-6}$ & -8.63321 & -8.63321 & $6.928269 \times 10^{-6}$ \\
1. & -2.71828 & -2.71828 & $1.8404898 \times 10^{-6}$ & -10.8731 & -10.8731 & $9.029439 \times 10^{-6}$ \\
\hline
\end{tabular}

Table 3: The numerical results of Example 3.1.

\begin{tabular}{lcccccc}
\hline$x$ & $u_{T}^{(3)}(x)$ & $u_{500}^{(3)}(x)$ & $\left|u_{T}^{(3)}(x)-u_{500}^{(3)}(x)\right|$ & $u_{T}^{(4)}(x)$ & $u_{500}^{(4)}(x)$ & $\left|u_{T}^{(4)}(x)-u_{500}^{(4)}(x)\right|$ \\
\hline 0 & -3 & -3.01001 & 0.010006 & -8 & -3.01001 & 4.98999 \\
0.2 & -4.93447 & -4.93447 & $8.518423 \times 10^{-9}$ & -11.53 & -11.5489 & 0.0188419 \\
0.4 & -7.69782 & -7.69782 & $5.477735 \times 10^{-8}$ & -16.3504 & -16.3771 & 0.0266921 \\
0.6 & -11.5887 & -11.5887 & $1.335948 \times 10^{-8}$ & -22.8858 & -22.9229 & 0.0370827 \\
0.8 & -17.0031 & -17.0031 & $1.724353 \times 10^{-8}$ & -31.6917 & -31.7423 & 0.050624 \\
1. & -24.4645 & -24.4645 & $2.771117 \times 10^{-13}$ & -43.4925 & $3.021444 \times 10^{-16}$ & 43.4925 \\
\hline
\end{tabular}

Table 4: The observed maximum errors of Example 3.2.

\begin{tabular}{lcccc}
\hline Method & $n=16$ & $n=32$ & $n=64$ & $n=128$ \\
\hline Our method & $1.18 \times 10^{-3}$ & $5.47 \times 10^{-4}$ & $2.62 \times 10^{-4}$ & $1.29 \times 10^{-4}$ \\
Nonpolynomial spline [11] & $7.12 \times 10^{-4}$ & $4.05 \times 10^{-4}$ & $2.22 \times 10^{-4}$ & $1.15 \times 10^{-4}$ \\
Quartic spline [12] & $1.15 \times 10^{-3}$ & $5.32 \times 10^{-4}$ & $2.56 \times 10^{-4}$ & $1.26 \times 10^{-4}$ \\
Finite difference [13] & $1.96 \times 10^{-4}$ & $4.89 \times 10^{-5}$ & $1.22 \times 10^{-5}$ & $3.06 \times 10^{-6}$ \\
Cubic spline [14] & $1.23 \times 10^{-3}$ & $5.53 \times 10^{-4}$ & $2.61 \times 10^{-4}$ & $1.27 \times 10^{-4}$ \\
Colloc. quantic spline [15] & $1.26 \times 10^{-3}$ & $5.60 \times 10^{-4}$ & $3.10 \times 10^{-4}$ & $1.67 \times 10^{-4}$ \\
Finite difference [16] & $6.89 \times 10^{-3}$ & $7.11 \times 10^{-3}$ & $7.27 \times 10^{-3}$ & $7.36 \times 10^{-3}$ \\
Quartic B spline [17] & $1.13 \times 10^{-3}$ & $5.30 \times 10^{-4}$ & $5.52 \times 10^{-4}$ & $1.23 \times 10^{-4}$ \\
\hline
\end{tabular}

Table 5: The numerical results of Example 3.3.

\begin{tabular}{lcccccc}
\hline$x$ & $u_{T}(x)$ & $u_{100}(x)$ & $\left|u_{T}(x)-u_{100}(x)\right|$ & $\left|u_{T}^{\prime}(x)-u_{100}^{\prime}(x)\right|$ & $\left|u_{T}^{\prime \prime}(x)-u_{100}^{\prime \prime}(x)\right|$ & $\left|u_{T}^{\prime \prime \prime}(x)-u_{100}^{\prime \prime \prime}(x)\right|$ \\
\hline 0 & 0 & 0 & 0 & $2.11892 \times 10^{-5}$ & $5.34582 \times 10^{-5}$ & $2.49157 \times 10^{-2}$ \\
0.1 & 0.110517 & 0.110518 & $1.06299 \times 10^{-6}$ & $6.59012 \times 10^{-6}$ & $4.42396 \times 10^{-6}$ & $2.85088 \times 10^{-4}$ \\
0.3 & 0.404958 & 0.404962 & $4.25342 \times 10^{-6}$ & $3.27006 \times 10^{-5}$ & $2.16465 \times 10^{-4}$ & $1.15677 \times 10^{-3}$ \\
0.5 & 0.824361 & 0.824376 & $1.51582 \times 10^{-5}$ & $7.46045 \times 10^{-5}$ & $1.67531 \times 10^{-4}$ & $8.58014 \times 10^{-4}$ \\
0.7 & 1.40963 & 1.40966 & $3.24736 \times 10^{-5}$ & $9.40379 \times 10^{-5}$ & $3.75442 \times 10^{-5}$ & $5.12073 \times 10^{-4}$ \\
0.8 & 1.78043 & 1.78047 & $4.20348 \times 10^{-5}$ & $9.71987 \times 10^{-5}$ & $3.89027 \times 10^{-5}$ & $5.59346 \times 10^{-4}$ \\
0.9 & 2.21364 & 2.21369 & $5.20699 \times 10^{-5}$ & $1.05201 \times 10^{-4}$ & $1.40742 \times 10^{-4}$ & $1.08743 \times 10^{-3}$ \\
1. & 2.71828 & 2.71835 & $6.36065 \times 10^{-5}$ & $1.27213 \times 10^{-4}$ & $1.9082 \times 10^{-4}$ & $1.07270 \times 10^{-2}$ \\
\hline
\end{tabular}


Table 6: The numerical results of Example 3.4, $p=q=\beta=1, \alpha=2, k=2, n=20$.

\begin{tabular}{cccccccc}
\hline$x$ & $u_{T}$ & $u_{20}$ & $\left|u_{T}-u_{20}\right|$ & $u_{T}^{\prime}$ & $u_{20}^{\prime}$ & $\left|u_{T}^{\prime}-u_{20}^{\prime}\right|$ & $\left|u_{T}^{\prime \prime}-u_{20}^{\prime \prime}\right|$ \\
\hline 0.08 & -0.0286650 & -0.0286650 & $5.20529 \times 10^{-9}$ & 0.0273871 & 0.0273873 & $2.32501 \times 10^{-7}$ & $7.75366 \times 10^{-6}$ \\
0.16 & -0.0255710 & -0.0255710 & $6.94285 \times 10^{-9}$ & 0.0490631 & 0.0490632 & $7.15527 \times 10^{-8}$ & $2.10654 \times 10^{-6}$ \\
0.24 & -0.0209523 & -0.0209523 & $9.70246 \times 10^{-9}$ & 0.0655842 & 0.0655842 & $1.41703 \times 10^{-8}$ & $5.37958 \times 10^{-7}$ \\
0.32 & -0.0152036 & -0.0152035 & $9.39437 \times 10^{-9}$ & 0.0773741 & 0.0773741 & $1.84526 \times 10^{-8}$ & $3.37592 \times 10^{-7}$ \\
0.48 & -0.0017588 & -0.0017588 & $1.71495 \times 10^{-9}$ & 0.0878568 & 0.0878567 & $8.12846 \times 10^{-8}$ & $4.48111 \times 10^{-7}$ \\
0.56 & 0.00525584 & 0.00525583 & $6.04974 \times 10^{-9}$ & 0.0868185 & 0.0868184 & $1.11842 \times 10^{-7}$ & $4.02874 \times 10^{-7}$ \\
0.64 & 0.0120206 & 0.0120205 & $1.65310 \times 10^{-8}$ & 0.0815939 & 0.0815937 & $1.53109 \times 10^{-7}$ & $6.25311 \times 10^{-7}$ \\
0.72 & 0.0181958 & 0.0181957 & $3.10494 \times 10^{-8}$ & 0.0720489 & 0.0720486 & $2.13478 \times 10^{-7}$ & $9.11482 \times 10^{-7}$ \\
0.88 & 0.0273346 & 0.0273346 & $8.10037 \times 10^{-8}$ & 0.0389010 & 0.0389005 & $4.47861 \times 10^{-7}$ & $1.32613 \times 10^{-6}$ \\
0.96 & 0.0295066 & 0.0295065 & $1.25247 \times 10^{-7}$ & 0.0144477 & 0.0144469 & $8.40832 \times 10^{-7}$ & $9.84025 \times 10^{-6}$ \\
\hline
\end{tabular}

Table 7: The numerical results of Example 3.5.

\begin{tabular}{lccccccc}
\hline$x$ & $u_{T}$ & $u_{20}$ & $\left|u_{T}-u_{20}\right|$ & $u_{T}^{\prime}$ & $u_{20}^{\prime}$ & $\left|u_{T}^{\prime}-u_{20}^{\prime}\right|$ & $\left|u_{T}^{\prime \prime}-u_{20}^{\prime \prime}\right|$ \\
\hline 0.08 & 0.000256 & 0.00025618 & $1.87335 \times 10^{-7}$ & 0.0096 & 0.009597 & $3.13184 \times 10^{-6}$ & $1.14007 \times 10^{-5}$ \\
0.16 & 0.002048 & 0.0020481 & $1.72967 \times 10^{-7}$ & 0.0384 & 0.038399 & $8.39162 \times 10^{-7}$ & $2.89347 \times 10^{-5}$ \\
0.24 & 0.006912 & 0.0069121 & $1.38265 \times 10^{-7}$ & 0.0864 & 0.0864 & $1.89713 \times 10^{-7}$ & $1.24163 \times 10^{-5}$ \\
0.32 & 0.016384 & 0.0163841 & $1.13116 \times 10^{-7}$ & 0.1536 & 0.1536 & $2.05885 \times 10^{-7}$ & $5.02804 \times 10^{-7}$ \\
0.4 & 0.032000 & 0.0320001 & $9.39829 \times 10^{-8}$ & 0.2400 & 0.2400 & $2.21979 \times 10^{-7}$ & $1.40284 \times 10^{-6}$ \\
0.48 & 0.055296 & 0.0552961 & $7.77508 \times 10^{-8}$ & 0.3456 & 0.3456 & $1.93052 \times 10^{-7}$ & $5.56607 \times 10^{-7}$ \\
0.56 & 0.087808 & 0.0878081 & $6.38527 \times 10^{-8}$ & 0.4704 & 0.4704 & $1.60088 \times 10^{-7}$ & $3.15123 \times 10^{-7}$ \\
0.64 & 0.131072 & 0.131072 & $5.21361 \times 10^{-8}$ & 0.6144 & 0.6144 & $1.33773 \times 10^{-7}$ & $3.10240 \times 10^{-7}$ \\
0.72 & 0.186624 & 0.186624 & $4.23781 \times 10^{-8}$ & 0.7776 & 0.7776 & $1.13440 \times 10^{-7}$ & $1.75032 \times 10^{-7}$ \\
0.8 & 0.256000 & 0.256000 & $3.44071 \times 10^{-8}$ & 0.9600 & 0.9600 & $9.20785 \times 10^{-8}$ & $3.49872 \times 10^{-7}$ \\
0.88 & 0.340736 & 0.340736 & $2.78679 \times 10^{-8}$ & 1.1616 & 1.1616 & $4.41977 \times 10^{-8}$ & $3.00596 \times 10^{-8}$ \\
0.96 & 0.442368 & 0.442368 & $2.08726 \times 10^{-8}$ & 1.3824 & 1.3824 & $2.08167 \times 10^{-8}$ & $9.08609 \times 10^{-6}$ \\
\hline
\end{tabular}

Example 3.4. Considering the following singular third-order three points boundary value problems with nonlinear condition

$$
\begin{gathered}
u^{\prime \prime \prime}(x)+\frac{p}{x^{\alpha}} u^{\prime \prime}(x)-\frac{k^{2}}{x^{\alpha}(1-x)^{\beta}} u^{\prime}(x)+\frac{q \sin (x)}{x^{\alpha}(1-x)^{\beta}} u(x)=f(x), \quad 0<x<1, \\
u^{\prime}(1)=0, \quad 2 u^{\prime}(0)=\left(u^{\prime \prime}(0)\right)^{2}, \quad u\left(\frac{1}{2}\right)=0 .
\end{gathered}
$$

We determine $f(x)$ to get the true solution, given by $u_{T}(x)=r(k(2 x-1)-2 \sinh (k x)+$ $2(\cosh (k x)) \tanh (k / 2)) / 2 k^{3}$. The numerical results are presented in Table 6. 
Example 3.5. Considering the following boundary value problems with nonlinear condition:

$$
\begin{gathered}
u^{\prime \prime \prime}(x)-\frac{1}{\sqrt{1+x}} u^{\prime}(x)+2 u(x)=f(x), \\
u(0)=u^{\prime}(0)=0, \quad\left(u^{\prime}(1)\right)^{2}=2 \int_{0}^{1} u(x) d x .
\end{gathered}
$$

We determine $f(x)$ to get the true solution, given by $u_{T}(x)=(1 / 2) x^{3}$. The numerical results are presented in Table 7.

\section{Conclusions and Remarks}

In this work, we present an algorithm for solving third-order mixed boundary value problems (BVPs) based on the reproducing kernel method. The method can be generalized to get reproducing kernel of problem with linear conditions. All computations are performed by the Mathematica 7.0 software package.

\section{Acknowledgments}

This paper is supported by the Natural Science Foundation of Inner Mongolia (no. 2009MS0103) and the project of Inner Mongolia University of Technology (no. ZS201036).

\section{References}

[1] J. Rashidinia and M. Ghasemi, "B-spline collocation for solution of two-point boundary value problems," Journal of Computational and Applied Mathematics, vol. 235, no. 8, pp. 2325-2342, 2011.

[2] H. N. Caglar, S. H. Caglar, and E. H. Twizell, "The numerical solution of third-order boundary-value problems with fourth-degree B-spline functions," International Journal of Computer Mathematics, vol. 71, no. 3, pp. 373-381, 1999.

[3] A. Khan and T. Aziz, "The numerical solution of third-order boundary-value problems using quintic splines," Applied Mathematics and Computation, vol. 137, no. 2-3, pp. 253-260, 2003.

[4] I. A. Tirmizi, E. H. Twizell, and Siraj-Ul-Islam, "A numerical method for third-order non-linear boundary-value problems in engineering," International Journal of Computer Mathematics, vol. 82, no. 1, pp. 103-109, 2005.

[5] M. Tatari and M. Dehghan, "The use of the Adomian decomposition method for solving multipoint boundary value problems," Physica Scripta, vol. 73, no. 6, pp. 672-676, 2006.

[6] M. Haque, M. H. Baluch, and M. F. N. Mohsen, "Solution of multiple point nonlinear boundary value problems by method of weighted residuals," International Journal of Computer Mathematics, vol. 18, no. 3, pp. 341-354, 1986.

[7] Y.-1. Wang and L. Chao, "Using reproducing kernel for solving a class of partial differential equation with variable-coefficients," Applied Mathematics and Mechanics, vol. 29, no. 1, pp. 129-137, 2008.

[8] Y. L. Wang, Z. Y. Li, Y. Cao, and X. H. Wan, "A new method for solving a class of mixed boundary value problems with singular coefficient," Applied Mathematics and Computation, vol. 217, no. 6, pp. 2768-2772, 2010.

[9] Z. Chen and Z.-J. Chen, "The exact solution of system of linear operator equations in reproducing kernel spaces," Applied Mathematics and Computation, vol. 203, no. 1, pp. 56-61, 2008.

[10] Y. L. Wang, C. L. Temuer, and J. Pang, "New algorithm for second-order boundary value problems of integro-differential equation," Journal of Computational and Applied Mathematics, vol. 229, no. 1, pp. $1-6,2009$. 
[11] S. Islam, S. I. A. Tirmizi, and M. A. Khan, "Non-polynomial splines approachto the solution of asystem of third-order boundary-value problems," Applied Mathematics Computation, vol. 168, no. 1, pp. 125$163,2005$.

[12] M. A. Noor and E. E. Al-Said, "Quartic splines solutions of third-order obstacle problems," Applied Mathematics and Computation, vol. 153, no. 2, pp. 307-316, 2004.

[13] E. A. Al-Said, "Numerical solutions for system of third-order boundary value problems," International Journal of Computer Mathematics, vol. 78, no. 1, pp. 111-121, 2001.

[14] E. A. Al-Said and M. A. Noor, "Cubic splines method for a system of third-order boundary value problems," Applied Mathematics and Computation, vol. 142, no. 2-3, pp. 195-204, 2003.

[15] M. A. Noor and A. K. Khalifa, "A numerical approach for odd-order obstacle problems," International Journal of Computer Mathematics, vol. 54, no. 1, pp. 109-116, 1994.

[16] E. A. Al-Said, M. A. Noor, and A. K. Khalifa, "Finite difference scheme for variational inequalities," Journal of Optimization Theory and Applications, vol. 89, no. 2, pp. 453-459, 1996.

[17] F. Gao and C.-M. Chi, "Solving third-order obstacle problems with quartic B-splines," Applied Mathematics and Computation, vol. 180, no. 1, pp. 270-274, 2006. 


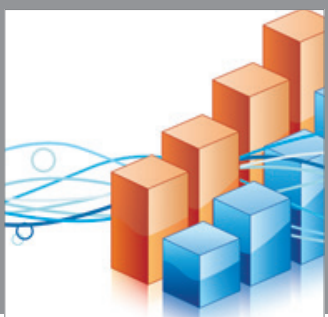

Advances in

Operations Research

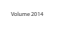

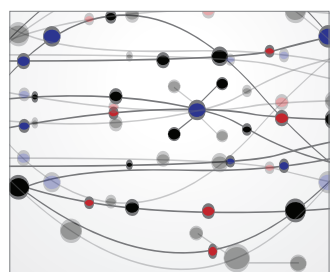

\section{The Scientific} World Journal
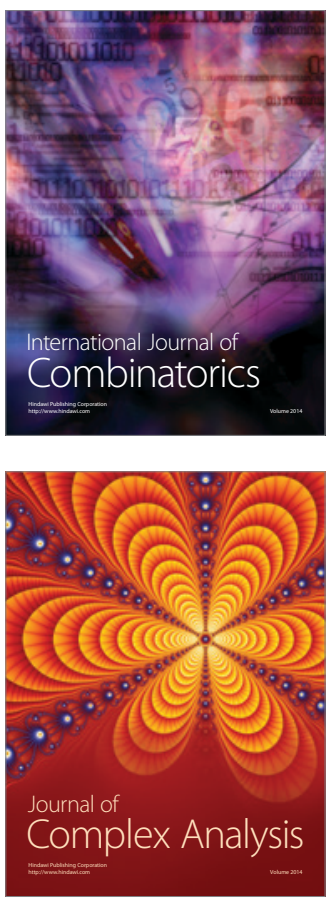

International Journal of

Mathematics and

Mathematical

Sciences
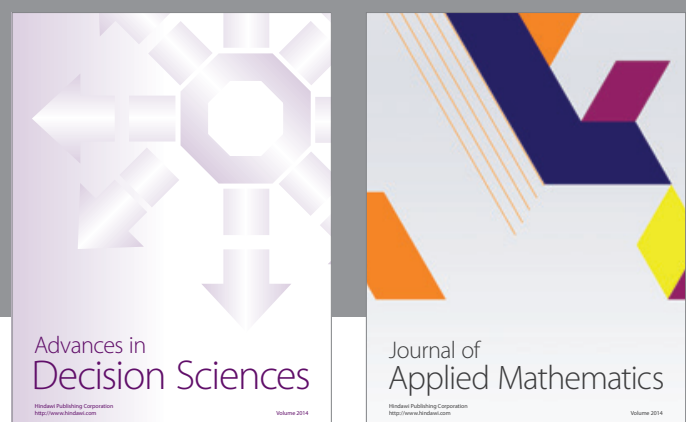

Journal of

Applied Mathematics
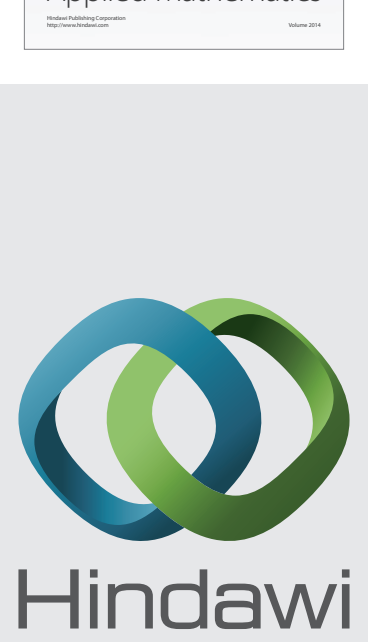

Submit your manuscripts at http://www.hindawi.com
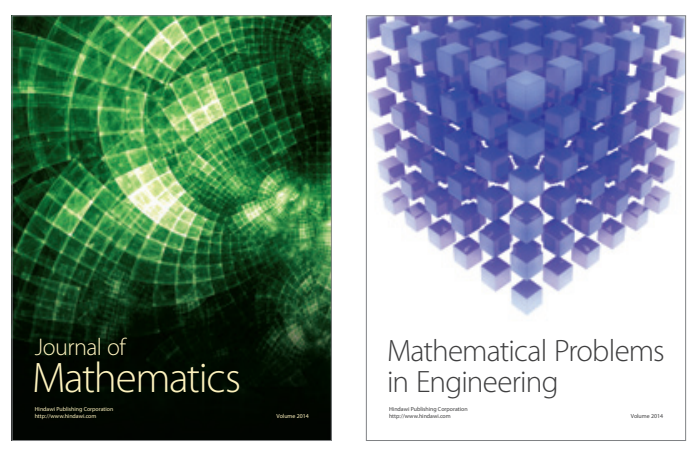

Mathematical Problems in Engineering
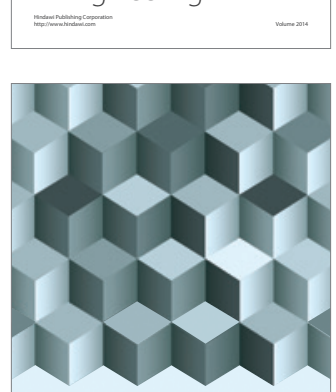

Journal of

Function Spaces
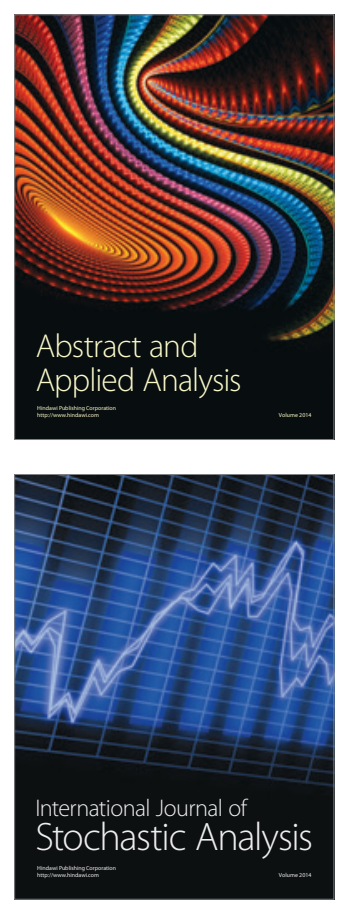

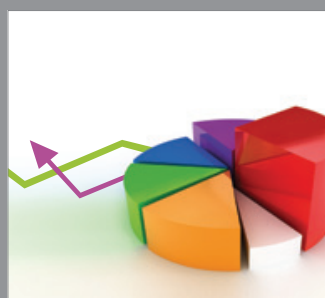

ournal of

Probability and Statistics

Promensencen
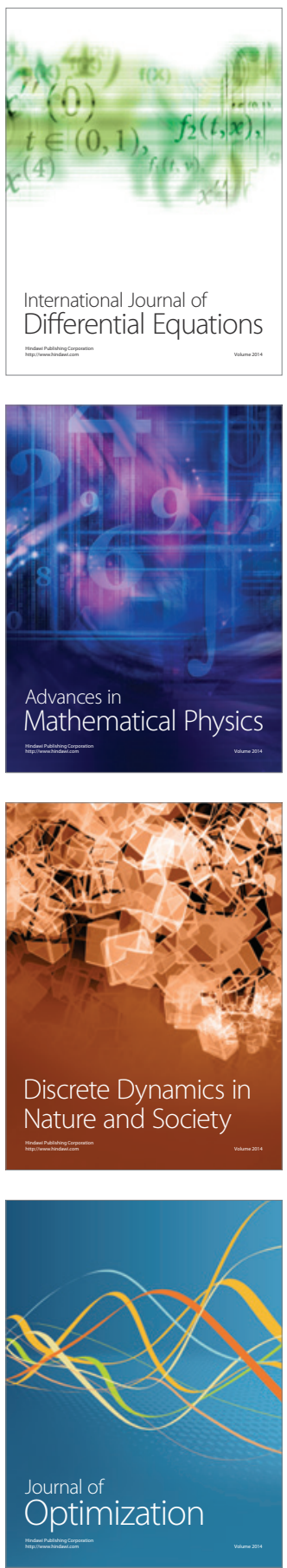